\title{
Extreme statistics of the excitations in the random transverse Ising chain
}

\author{
István A. Kovács $\odot,{ }^{1,2, *}$ Tamás Pető, ${ }^{3, \dagger}$ and Ferenc Iglói $\circledast^{4,3, \hbar}$ \\ ${ }^{1}$ Department of Physics and Astronomy, Northwestern University, Evanston, Illinois 60208, USA \\ ${ }^{2}$ Northwestern Institute on Complex Systems, Northwestern University, Evanston, Illinois 60208, USA \\ ${ }^{3}$ Institute of Theoretical Physics, University of Szeged, H-6720 Szeged, Hungary \\ ${ }^{4}$ Wigner Research Centre for Physics, Institute for Solid State Physics and Optics, H-1525 Budapest, P.O. Box 49, Hungary
}

(Received 12 March 2021; accepted 23 June 2021; published 11 August 2021)

\begin{abstract}
In random quantum magnets, like the random transverse Ising chain, low energy excitations are localized in rare regions and there are only weak correlations between them. It is an open question whether these correlations are relevant in the sense of the renormalization group. To answer this question, we calculate the distribution of the excitation energy of the random transverse Ising chain in the disordered Griffiths phase with high numerical precision by the strong disorder renormalization group method and-for shorter chains-by free-fermion techniques. Asymptotically, the two methods give identical results, which are well fitted by the Fréchet limit law of the extremes of independent and identically distributed random numbers. Considering the finite size corrections, the two numerical methods give very similar results, but these differ from the correction term for uncorrelated random variables, indicating that the weak correlations between low-energy excitations in random quantum magnets are relevant.
\end{abstract}

DOI: 10.1103/PhysRevResearch.3.033140

\section{INTRODUCTION}

Many-body systems in the presence of quenched disorder have unusual dynamical properties due to rare-region effects. In these systems - due to extreme fluctuations of strong couplings - domains are formed, which can remain locally ordered even in the paramagnetic phase. The relaxation time $\tau$, associated with turning the spins in such domains, can be extremely large and it has no upper limit in the thermodynamic limit. These types of Griffiths singularities are responsible for nonanalytical behavior of several physical quantities (susceptibility, specific heat, autocorrelation function) in the so called Griffiths phase, which is an extended part of the paramagnetic phase [1].

In random quantum systems, Griffiths effects are stronger than in classical ones, manifested in the power-law decay of the autocorrelation function, as well as power-law singularities of the susceptibility and the specific heat at low temperatures [2,3]. In random quantum magnets with discrete symmetry, such as in the random transverse Ising model (RTIM), the low-energy excitations are localized and their properties can be successfully studied by the so called strong disorder renormalization group (SDRG) method [4]. As initiated by Ma, Dasgupta, and Hu [5], and further developed by

\footnotetext{
*istvan.kovacs@ northwestern.edu

†petotamas0@gmail.com

¥igloi.ferenc@wigner.hu
}

Published by the American Physical Society under the terms of the Creative Commons Attribution 4.0 International license. Further distribution of this work must maintain attribution to the author(s) and the published article's title, journal citation, and DOI.
Fisher [6], the SDRG is a local renormalization technique in which quantum and disorder fluctuations are treated simultaneously, and degrees of freedom with a large excitation energy are successively eliminated. In these random quantum magnets the SDRG method is expected to provide asymptotically exact results at the critical point, the properties of which are governed by an infinite disorder fixed point [7]. Here, the Griffiths phase is controlled by strong disorder fixed points, but there are some cases in which the SDRG describes the dynamical singularities asymptotically exactly [8-10], for a counterexample see Ref. [11].

In phenomenological descriptions, it is often assumed that the localized excitations in random quantum magnets are independent [12]. For example, distributions of low-energy excitations in these systems are well approximated by the Fréchet distributions [13], which represents the limit law of the extremes of independent and identically distributed (iid) random numbers. Recently, extreme value statistics (EVS) has been applied to several problems, we can mention earthquakes, tsunamis, extreme flooding, big wildfires, extremes of climate, stock market risks in finance, sport records, etc. [14-19]. A complete understanding of extreme value statistics is known for uncorrelated random numbers, in which case also the convergence to the limit laws is derived by mathematical [20] and renormalization group [21-24] (RG) methods. The iid limit distributions also apply to weakly correlated random numbers, while new types of limit distributions appear for strongly correlated cases [25].

It is an intriguing problem to what extent the localized excitations in random quantum magnets are independent? Are weak correlations between the rare regions manifested in some effects, such as in the form of finite-size corrections? In this paper, we address this question and consider the 
paradigmatic model, the RTIM in one dimension (1D), and calculate numerically the distribution of the first energy gap by the asymptotically exact SDRG method with high accuracy. For moderate $L$ system sizes, we use also free-fermion techniques [26-28] to calculate the gaps of the random samples exactly. The distribution of the gaps for finite $L$ are compared with an appropriate Fréchet distribution and their difference is analyzed through finite-size scaling. To check the potential form of numerical corrections, the same type of numerical test is repeated for iid random numbers too.

The rest of the paper is organized as follows. In Sec. II the RTIM model is introduced and known results are summarized. In Sec. III the basic results about the extremes of uncorrelated variables and their finite-size corrections are recapitulated. In Sec. IV the distributions of gaps of the RTIM are calculated and the finite-size corrections are compared with the analytical results of uncorrelated variables. To test the numerical accuracy we repeat this analysis for uncorrelated Kesten variables [29]. Our paper concludes with a discussion in the final section. The methods to calculate the excitation energy of the RTIM are described in the Appendix.

\section{MODEL AND KNOWN RESULTS}

Here, we consider the RTIM in 1D defined by the Hamiltonian

$$
\hat{H}=-\sum_{i=1}^{L-1} J_{i} \sigma_{i}^{z} \sigma_{i+1}^{z}-\sum_{i=1}^{L} h_{i} \sigma_{i}^{x},
$$

in terms of the $\sigma_{i}^{x, z}$ Pauli matrices at site $i$ and the nearest neighbor couplings $J_{i}>0$ and the transverse fields $h_{i}>0$ are taken from the distributions $\pi_{1}(J)$ and $\pi_{2}(h)$, respectively. Generally, we use open boundary conditions (OBCs) and work at zero temperature $T=0$.

In the thermodynamic limit $L \rightarrow \infty$, the control parameter is defined as [6]

$$
\delta=\frac{[\ln h]_{\mathrm{av}}-[\ln J]_{\mathrm{av}}}{\operatorname{var}(h)+\operatorname{var}(J)},
$$

where $[\cdots]_{\text {av }}$ denotes averaging over quenched disorder and $\operatorname{var}(x)$ stands for the variance of $x$. For $\delta<0(\delta>0)$ the system is in the ordered ferromagnetic (disordered paramagnetic) phase and at $\delta=0$ there is a random quantum critical point. According to SDRG calculations $[6,8,9]$ and numerical results [30,31] the critical behavior of the system is controlled by an infinite disorder fixed point [7]. For example, the energy scale, defined by the lowest gap $\varepsilon$, and the length scale are related as

$$
\ln \varepsilon \sim L^{1 / 2}, \quad \delta=0 .
$$

In the paramagnetic phase this relation is in a power-law form

$$
\varepsilon \sim L^{-z}, \quad \delta>0,
$$

which is due to Griffiths singularities. Here, the dynamical exponent $z=z(\delta)$ depends on the distance from the critical point and is given by the positive root of the equation [8-10]

$$
\left[\left(\frac{J}{h}\right)^{1 / z}\right]_{\mathrm{av}}=1,
$$

and in the vicinity of the critical point it diverges as $z \approx$ $1 /(2 \delta)$.

The distribution of the first gap has been calculated analytically through the SDRG method [32]

$$
P_{L}(\varepsilon ; z)=\frac{1}{z} u^{1 / z-1} \exp \left(-u^{1 / z}\right)
$$

in terms of $u=u_{0} \varepsilon(L / \xi)^{z}, \xi$ being the correlation length and $u_{0}$ is a constant, fixed by the choice of standardization, as discussed in the next section. This relation is valid for $L \gg \xi$ and for $\delta \ll 1$, in which limit $\xi \sim \delta^{-2}$. We observe that Eq. (6) is just the Fréchet distribution, see Eq. (19).

An approximate form of the distribution of the low-energy excitations can be obtained from the assumption that these excitations are localized and are due to extreme fluctuations of say $n \ll L$ consecutive strong bonds [12]. The probability to find such a strongly connected cluster in the system is given by $P_{L}(n) \sim L \exp (-a n)$. At the same time the excitation energy due to such a cluster is exponentially small: $\varepsilon \sim$ $\exp (-b n)$. Combining these expressions we obtain $P_{L}(\ln \varepsilon) \sim$ $L \varepsilon^{1 / z}$, with $z=b / a$ being the dynamical exponent in agreement with Eq. (4). Then, the cumulated distribution of the relaxation times $\tau \sim 1 / \varepsilon$ is obtained in this approach as

$$
\mu(\tau) \approx 1-A \tau^{-1 / z}, \quad \tau \gg 1 .
$$

If we assume that the excitations are uncorrelated following the reasoning in Sec. III we arrive at the distribution in Eq. (6), which is calculated by the SDRG approach in the given limits.

In this paper, we aim to study this problem in more detail, considering the following points. (i) To calculate the distribution function through numerical implementation of the SDRG approach and to check if the result in Eq. (6) is valid in the entire Griffiths phase. (ii) To confirm the results of the SDRG calculation with the exact gaps obtained through free-fermion techniques. (iii) To check the form of the finite-size corrections of the two methods and to compare with the analytical result for the extremes of uncorrelated random variables.

\section{EXTREME STATISTICS OF UNCORRELATED VARIABLES}

Let us have a process of iid random numbers $u_{1}, u_{2}, \ldots, u_{N}$ and consider the maximum value $u=\max \left(u_{1}, u_{2}, \ldots, u_{N}\right)$. Each random variable is distributed by the same, so called parent distribution function $\rho(u)$ and the cumulative (or integrated) parent distribution is given by $\mu(u)=\int_{-\infty}^{u} \rho(t) d t$. The cumulative distribution of the maximum value $u$ is easy to compute:

$$
M_{N}^{\max }(u)=\operatorname{Prob}\left[u_{1} \leqslant u, u_{2} \leqslant u, \ldots, u_{N} \leqslant u\right]=\mu^{N}(u) .
$$

According to extreme value statistics (EVS) $M_{N}^{\max }(u)$ has a limit distribution for large $N$ and large $u$ in terms of the scaling combination $v=\left(u-b_{N}\right) / a_{N}$ as

$$
M_{N}(v)=M_{N}^{\max }\left(a_{N} v+b_{N}\right) \rightarrow M(v),
$$

and similarly for the extreme density $P_{N}(v)=d M_{N} / d v$,

$$
P_{N}(v)=a_{N} P_{N}^{\max }\left(a_{N} v+b_{N}\right) \rightarrow P(v) .
$$

Here, the $a_{N}$ and $b_{N}$ are free up to an additive constant, the value of which is fixed by different standardization conditions. 
For the analytical calculation the condition

$$
M(0)=P(0)=1 / e
$$

is convenient to use, whereas for analyzing numerical data it is often better to require

$$
\int_{-\infty}^{\infty} v P(v)=0, \quad \int_{-\infty}^{\infty} v^{2} P(v)=1,
$$

provided the second moment of the distribution exists. In this paper, we refer to Eqs. (11) and (12) as first and second standardization conditions, respectively.

\section{A. Renormalization group analysis}

In a renormalization group treatment [21,22], one starts with the invariance condition

$$
M(v)=M^{p}[a(p) v+b(p)] \equiv\left[\hat{R}_{p} M\right](v),
$$

which should be satisfied for any positive rational number $p$. The operator $\hat{R}_{p}$ acts on the parent integrated distribution as

$$
\left[\hat{R}_{p} \mu\right](v)=\mu^{p}[a(p) v+b(p)],
$$

and the first standardization condition in Eq. (11) requires

$$
\begin{gathered}
b(p)=\mu^{-1}\left(e^{-1 / p}\right), \\
a(p)=p \frac{d b(p)}{d p} .
\end{gathered}
$$

In the RG treatment, the invariance condition in Eq. (13) is interpreted as a fixed-point relation

$$
\left[\hat{R}_{p} M\right](v)=M(v),
$$

and its general solutions depend on the parameter $\gamma$ and are given in the form

$$
\begin{gathered}
M(v ; \gamma)=\exp \left[-(1+\gamma v)^{-1 / \gamma}\right], \\
P(v ; \gamma)=(1+\gamma v)^{-1 / \gamma-1} \exp \left[-(1+\gamma v)^{-1 / \gamma}\right],
\end{gathered}
$$

for $1+\gamma v \geqslant 0$. The parameter $\gamma$ is related to the large $u$ tail of the parent distribution and defines the different universality classes. For $\gamma>0$ the parent distribution is given by

$$
\mu(u) \approx 1-A u^{-1 / \gamma}, \quad u \gg 1,
$$

and represents the Fréchet universality class. For $\gamma=0$ the asymptotic approach of the cumulative parent distribution is faster than a power and represents the Gumbel universality class. Finally, for $\gamma<0$ the asymptotic maximum value of the integrated parent distribution is approached at a finite upper bound with a power $-1 / \gamma$ and the limit distribution is given by the Weibull distribution.

\section{B. Finite-size corrections}

To obtain the finite-size corrections in the RG treatment one should consider a perturbation at the fixed point having a small parameter $\varepsilon$ and introduce the perturbed distribution function

$$
\begin{aligned}
M(v ; \gamma, \varepsilon) & =M(v ; \gamma)+\varepsilon M_{1}(v ; \gamma) \\
& =M(v ; \gamma)+\varepsilon P(v ; \gamma) \psi(v) .
\end{aligned}
$$

Here, the correction function $\psi(v)$ satisfies the first standardization condition as

$$
\psi(0)=0, \quad \psi^{\prime}(0)=0, \quad \psi^{\prime \prime}(0)=-1,
$$

where the third condition sets the sign and scale of $\varepsilon$. The perturbed distribution function should satisfy the RG equation

$$
\left[\hat{R}_{p} M(\varepsilon)\right](v)=M^{p}[a(\varepsilon) v+b(\varepsilon) ; \varepsilon]=M\left(v ; \varepsilon^{\prime}\right) .
$$

Here, we expand $M(\varepsilon), a(\varepsilon)$, and $b(\varepsilon)$ in linear order in $\varepsilon$ and use the scaling law of the perturbation

$$
\varepsilon^{\prime} / \varepsilon=p^{\gamma^{\prime}},
$$

which defines the correction to scaling exponent $\gamma^{\prime}$. The correction to the scaling function $\psi\left(v ; \gamma, \gamma^{\prime}\right)$ depends also on $\gamma^{\prime}$ and is given in the first standardization condition as

$$
\psi\left(v ; \gamma, \gamma^{\prime}\right)=\frac{(1+\gamma v)+\gamma^{\prime} v-(1+\gamma v)^{\gamma^{\prime} / \gamma+1}}{\gamma^{\prime}\left(\gamma^{\prime}+\gamma\right)} .
$$

In a similar fashion, the probability density in Eq. (10) is extended by the perturbation

$$
P(v ; \gamma, \varepsilon)=P(v ; \gamma)+\varepsilon P_{1}\left(v ; \gamma, \gamma^{\prime}\right),
$$

and $P_{1}\left(v ; \gamma, \gamma^{\prime}\right)=d M_{1}\left(v ; \gamma, \gamma^{\prime}\right) / d v$ depends on the decay exponent of the parent distribution $\gamma$, as well as the correction exponent $\gamma^{\prime}$.

\section{Connection to the parent distribution}

In a practical calculation one starts with the parent distribution and calculates the effective decay exponent, which can be obtained as

$$
\gamma_{N}=\frac{d a_{N}}{d b_{N}},
$$

where $a_{N}$ and $b_{N}$ are defined in the transformation law in Eq. (9). The leading correction term is given by

$$
\varepsilon_{N}=\gamma-\gamma_{N} \propto N^{\gamma^{\prime}} .
$$

which is just the finite-size correction to the parameter $\gamma$. Regarding the Fréchet distribution, generally the parent distribution has a nonanalytical correction term

$$
\mu(u) \approx 1-A u^{-1 / \gamma}\left(1+B u^{-\omega}\right), \quad u \gg 1,
$$

and the finite-size corrections to $\gamma_{N}$ are of two types. There is an analytical correction with $\gamma_{\text {analytic }}^{\prime}=-1$ and a nonanalytic correction with $\gamma_{\text {nonanalytic }}^{\prime}=-\omega \gamma$. Thus, the leading correction exponent is given by

$$
\gamma^{\prime}=-\min (1, \omega \gamma) \text {. }
$$

\section{Exponential distribution}

In practical analysis of the data with a parent distribution in Eq. (20), it is convenient to use logarithmic variables $x=\ln u$, so that $e^{x-x_{0}}=1+\gamma v$. In this case, the parent distribution of $x$ is exponential and the extreme distribution is given by the Gumbel form

$$
M_{G}(x, \gamma)=\exp \left[-\exp \left(\frac{-x+x_{0}}{\gamma}\right)\right],
$$




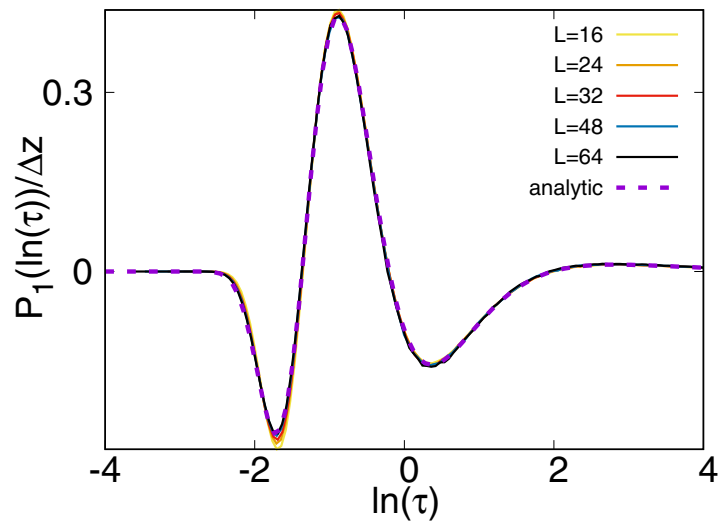

FIG. 1. Finite-size corrections to the distributions of the $\log$ extremes for a pure power parent distribution with the second standardization condition, see text.

and similarly for the probability density

$$
P_{G}(x, \gamma)=\frac{1}{\gamma} \exp \left(\frac{-x+x_{0}}{\gamma}\right) \exp \left[-\exp \left(\frac{-x+x_{0}}{\gamma}\right)\right],
$$

so that $M_{G}\left(x_{0}, \gamma\right)=1 / e$ and $P_{G}\left(x_{0}, \gamma\right)=1 /(e \gamma)$. For the Gumbel distribution, the finite-size correction term is given by $P_{1}\left(x ; 0, \gamma^{\prime}\right)$, see Eq. (26), and in the numerical examples the correction exponent is $\gamma^{\prime}=-1$. In this representation it is convenient to use the second standardization condition, in which case the correction terms are transformed [21]. For $\gamma=0$ and $\gamma^{\prime}=-1$ the correction to the probability density in the second standardization condition is given by [33]

$$
\begin{gathered}
P_{1}^{2^{\mathrm{nd}}}(x ; 0,-1)=a e^{-\tilde{x}-e^{-\tilde{x}}}\left[\left(e^{-\tilde{x}}-1\right)\left(1-x / a-e^{-\tilde{x}}\right)\right. \\
\left.-a^{-2}+e^{-\tilde{x}}\right],
\end{gathered}
$$

with $\tilde{x}=a x+b, a=\pi / \sqrt{6}$ and $b=\gamma_{E}=0.5772156649$ being the Euler-Mascheroni constant. As an illustration, we consider a pure power parent distribution $\mu(u)=1-$ $u^{-1 / \gamma}, u \geqslant 1$ and in Fig. 1 show the numerically calculated finite-size corrections to the distributions of the log extremes, denoted by $\ln (\tau)$, for $L=16,24,32,48$, and 64 with $\gamma=$ $z\left(h_{0}=3\right)$, see Eq. (36), in the second standardization condition. These are to be compared with the analytical result in Eq. (33), as in this case $\gamma^{\prime}=-1$. The agreement is next to perfect.

\section{NUMERICAL RESULTS}

In the numerical calculation the parameters of the Hamiltonian $J_{j}$ and $h_{j}$ are taken from boxlike distributions

$$
\begin{aligned}
& \pi_{1}(J)= \begin{cases}1 & \text { for } 0<J \leqslant J_{0}, \\
0 & \text { otherwise, }\end{cases} \\
& \pi_{2}(h)= \begin{cases}1 / h_{0} & \text { for } 0<h \leqslant h_{0}, \\
0 & \text { otherwise, }\end{cases}
\end{aligned}
$$

and we set the energy scale with $J_{0}=1$. In this case, the critical point of the RTIM is located at $h_{0}=1$, and in the disordered phase $\left(h_{0}>1\right)$, the dynamical exponent satisfies
TABLE I. Finite-size estimates of the dynamical exponent $z_{L}$ at $h_{0}=4$ calculated by the SDRG algorithm from the best fit of the Fréchet form in Eq. (32) and its difference from the asymptotically exact value $z=1.2112288988$. Exponent of a power-law fit $a$ and that of the logarithmic correction $\omega$. (See text.) In parentheses, the results obtained by free-fermion calculation are presented.

\begin{tabular}{lcccc}
\hline \hline$L$ & $z_{L}$ & $z-z_{L}$ & $a$ & $\omega$ \\
\hline 32 & 1.102788 & 0.108441 & & \\
$(32)$ & $(1.119570)$ & $(0.091659)$ & & \\
64 & 1.142667 & 0.068562 & 0.6614 & 1.4081 \\
$(64)$ & $(1.152473)$ & $(0.058756)$ & $(0.6415)$ & $(1.3627)$ \\
128 & 1.169523 & 0.041706 & 0.7172 & 1.3724 \\
256 & 1.186572 & 0.024657 & 0.7583 & 1.3405 \\
512 & 1.196923 & 0.014306 & 0.7854 & 1.3388 \\
\hline \hline
\end{tabular}

the equation

$$
\left(1-z^{-2}\right) h_{0}^{1 / z}=1,
$$

see Eq. (5). In the vicinity of the critical point $z$ diverges as $z \approx \frac{1}{\ln h_{0}}, h_{0} \rightarrow 1^{+}$, whereas for large $h_{0}$ it approaches 1 as $z \approx 1+\frac{1}{2 h_{0}}, h_{0} \rightarrow \infty$.

We considered three specific points of the paramagnetic phase, having the dynamical exponents

$$
z= \begin{cases}1.7476551, & h_{0}=2 \\ 1.3354200, & h_{0}=3 \\ 1.2112289, & h_{0}=4\end{cases}
$$

In the free-fermion calculation of the gaps, the lengths of the systems were $L=16,24,32,48$, and 64 , whereas with the SDRG method larger systems up to $L=512$ are treated. In all cases $10^{10}$ independent samples are investigated.

\section{A. SDRG gaps and the Fréchet distribution}

We start by analyzing the data generated by the SDRG method and presenting the distributions of the log gaps in Fig. 2, for different sizes. To test their relation with the Fréchet distribution, we calculated in each case the best fit of the analytical function in Eq. (32), having the dynamical exponent $z_{L} \equiv \gamma_{L}$ and the position of the maximum $x_{0}=x_{0}(L)$ as fitting parameters. The resulting best fits are also shown in Fig. 2.

As seen in Fig. 2, the Fréchet distribution describes the numerical data well and the difference between the numerical points and the fitting curve is decreasing for increasing values of $L$. At a given value of $h_{0}$ the fitted value of the dynamical exponent $z_{L}$ has a small variation with the size, and approaches the exact asymptotic value, as given in Eq. (35). At the value $h_{0}=4$, this is illustrated in Table I, where the second column shows the fitted, finite size values of $z_{L}$, while the third column shows the difference from the exact value. As a first step, we used a power-law form $z-z_{L} \sim L^{-a}$ to fit the finite-size corrections, and estimates for the exponent $a$ are calculated through two-point fit. These are listed in the fourth column of the table and have a slow convergence, which generally indicates a strong correction to scaling term. Having 

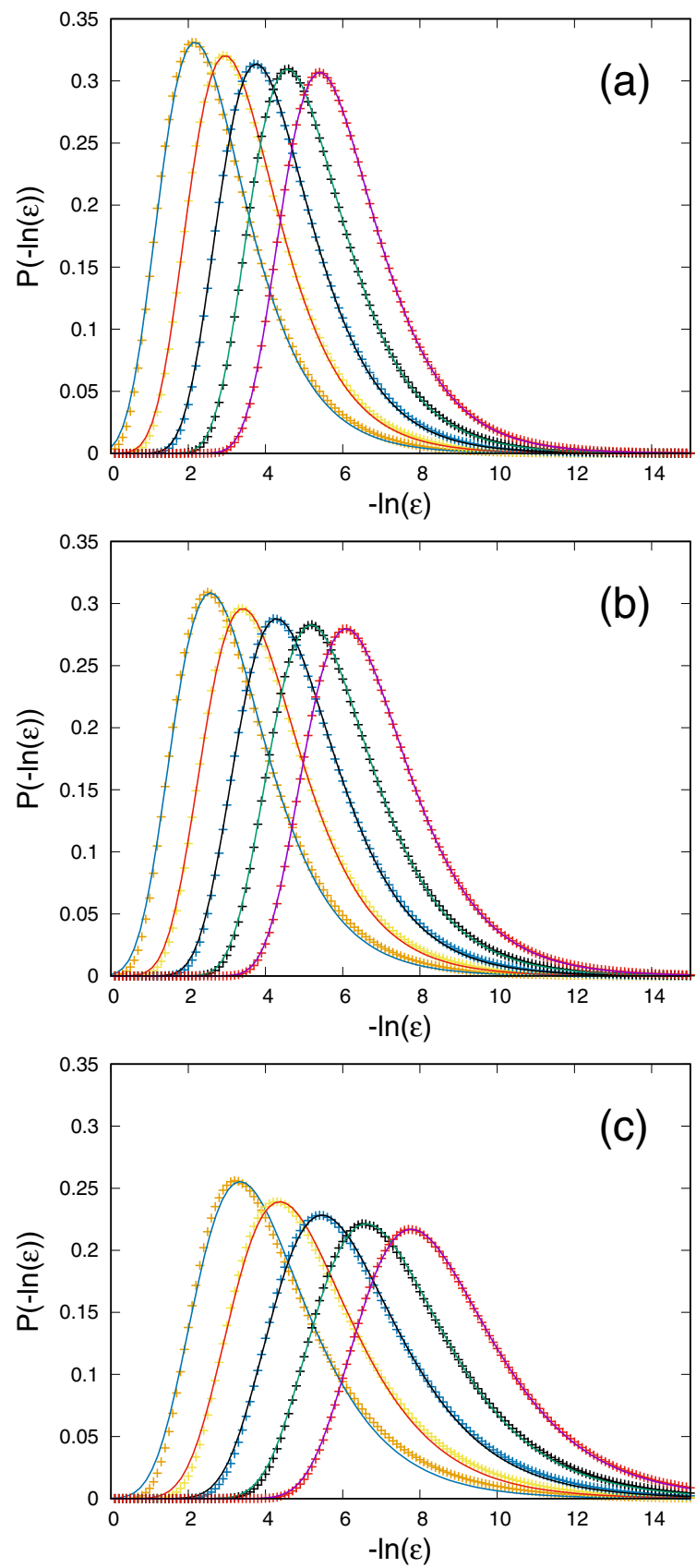

FIG. 2. Distribution of the log energy gaps, calculated by the SDRG algorithm in the Griffiths phase with $h_{0}=4$ (a), $h_{0}=3$ (b), and $h_{0}=2$ (c), for finite chains of lengths $L=32,64,128,256$, and 512, from left to right. The best fit of the Fréchet distribution is indicated by full lines.

this possibility in mind, we have used another functional form

$$
z-z_{L} \sim \frac{\ln ^{\omega} L}{L} .
$$

In this case, the effective exponents for $\omega$ are also calculated through two-point fit and are presented in the fifth column of the table. This type of fitting turned out to be more stable, the effective exponents seem to converge to $\omega \approx 1.33$.

We have repeated the same analysis at the other two points of the Griffiths phase. In both cases, the log-correction form is found to provide a better fit, with the correction exponents $\omega \approx 1.83$ at $h_{0}=2$ and $\omega \approx 1.50$ at $h_{0}=3$.

Most importantly, at a given value of $h_{0}$ in Fig. 2, the distributions are shifted with increasing size, and according to Eq. (4) the position of the maximum is expected to follow the rule: $x_{0}(L) \approx$ const. $+z_{L} \ln L$. Comparing the position of the maximum of the distribution at two sizes one can obtain estimates for the dynamical exponent as $z(L, 2 L)=\left[x_{0}(2 L)-\right.$ $\left.x_{0}(L)\right] / \ln 2$. We have checked that generally $z_{L}<z(L, 2 L)<$ $z_{2 L}$ and these estimates approach the asymptotic exact value with the same type of corrections as noticed for the case of $z_{L}$ in the previous paragraph.

We note that the analysis of the data obtained by freefermion calculation of the gap gives similar results. In Table I we have included the numerical results in parentheses for the two smallest sizes, but in this case the asymptotic region of the effective exponents $z_{L}$ is more remote. The logarithmic correction of scaling in Eq. (37) is quite unusual, so some words about its possible origin will be in order. First, we mention that at this point the analytical and the nonanalytical corrections have the same $1 / L$ dependence, see Eq. (29), and this type of interplay could result in a logarithmic correction, cf. Ref. [34]. A second argument is related to the fact that the linear extension of regions with a large size $l$ scales as $l \sim \ln L$. Even if the dominant rare regions had a finite extent, as considered in Ref. [35], their possible positions in the chain are constrained by the other more extended clusters, potentially resulting in a logarithmic multiplicative factor in Eq. (37).

\section{B. Finite-size corrections to the gap distributions}

We considered the gaps calculated by SDRG iteration for $L=24,32,48,64,96$, and 128 and for comparison we calculated those by free-fermion techniques as well, for shorter chains with $L=16,24,32,48$, and 64, except for $h_{0}=4$, where the corrections are the smallest and we went up to $L=48$. We analyzed the finite-size scaling of distributions in two different ways. First, we used the first standardization condition in Eq. (11) and calculated the difference with the Fréchet extreme distribution, with an effective $z_{L}$, calculated from the relations below Eq. (32). This difference is then rescaled by a factor $\Delta z=z-z_{L}$, and the results for $h_{0}=2$, 3 , and 4 are drawn in Fig. 3. In these figures the analytical results calculated for iid random numbers in Eq. (25) with $\gamma=z$ and $\gamma^{\prime}=-1$ are also presented. Here, the correction to scaling exponent $\gamma^{\prime}=-1$ corresponds to the expected scaling form in Eq. (37), which is obtained through numerical analysis of the data in Table I. According to Fig. 3, we can draw the following conclusions. (i) The scaled finite-size difference of the distribution function seems to approach a limiting curve for large $L$, which depends on $z\left(h_{0}\right)$. (ii) At a given $z\left(h_{0}\right)$ the limiting curves are similar (if not identical) for the SDRG and the free-fermion data. (iii) The convergence to this limit curve is slow, much slower than that of the iid random numbers, see later in Sec. IV C and the figures in the third column of Fig. 3. This slow convergence is probably related to the logarithmic correction to the scaling of the dynamical exponent. (iv) Finally, the expected limit curve of the numerical distribution of the finite-size correction differs from that of the analytical 

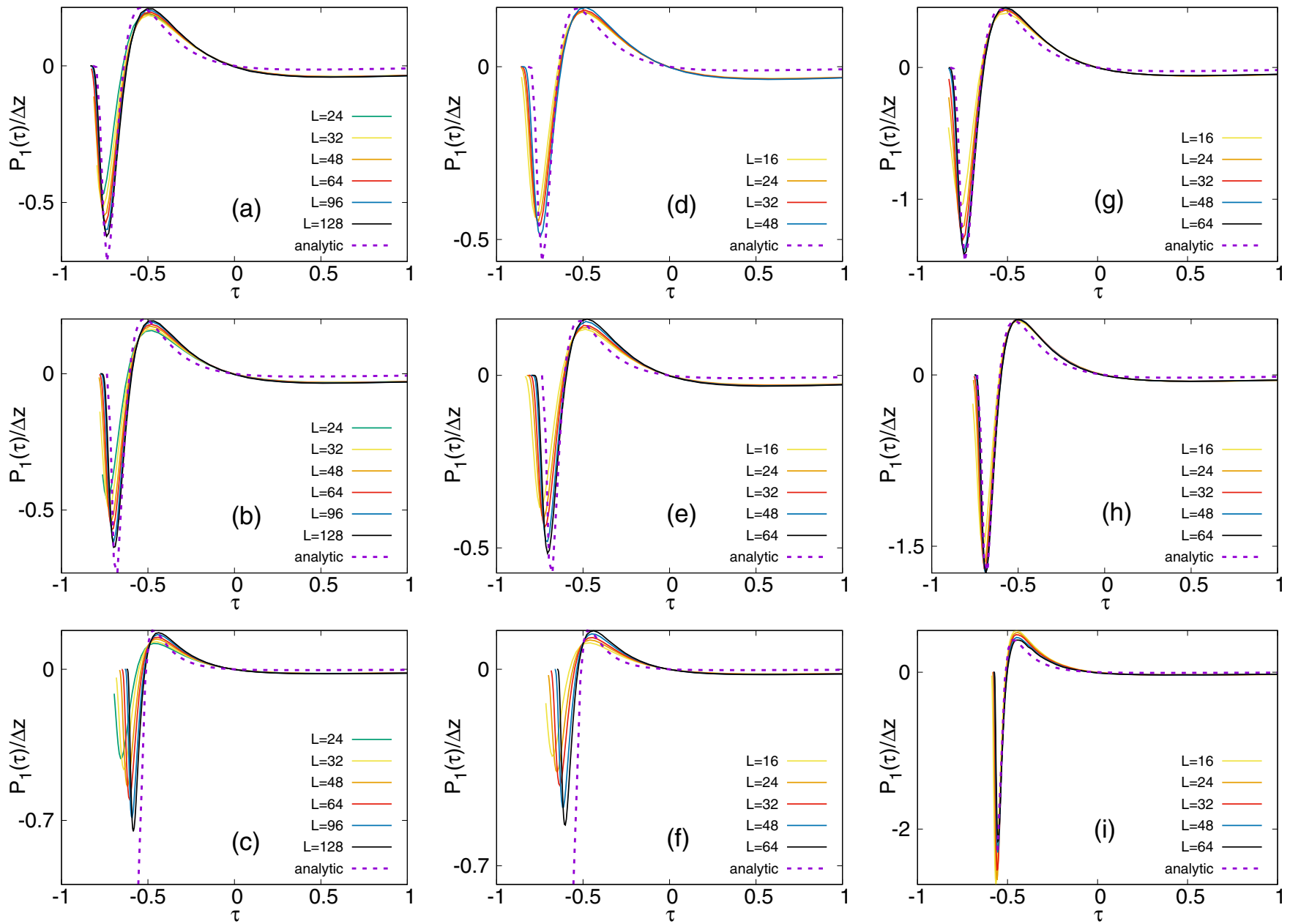

FIG. 3. Finite-size corrections to the Fréchet distribution for the inverse gaps $\tau=1 / \varepsilon$ in the RTIM at different sizes $L$ calculated through SDRG iterations [left panels (a), (b), and (c)], and by the free-fermion method [middle panels (d), (e), and (f)] with the first standardization in Eq. (11) and compared with the analytical results with $\gamma=z\left(h_{0}\right)$ and $\gamma^{\prime}=-1$ (dashed lines). In the right panels [(g), (h), and (i)] the maximum of uncorrelated Kesten random variables are shown, having the same scaling exponents, see text. The scaling exponents $z\left(h_{0}\right)$ correspond to $h_{0}=4$ [first row (a), (d), and (g)], $h_{0}=3$ [second row (b), (e), and (h)], and $h_{0}=2$ [third row (c), (f), and (i)], see Eq. (35). Note that by requesting the first standardization condition in Eq. (22) the numerical curves are shifted to the same reference point and scaled to a master curve by dividing with $\Delta z=z-z_{L}$.

result calculated for iid random numbers (having identical parameter $z$ ). Even though the overall shapes of the curves are similar, there are noticeable differences. In particular, the low-energy part is more strongly represented in the numerical curves, which can be interpreted as the reduction of the value of the gap due to small, but relevant correlations between rare regions.

We have repeated the analysis with the distribution of the log gaps and using the second standardization condition in Eq. (12). The resulting finite-size corrections of the distributions are shown in Fig. 4 together with the analytical curves for iid random numbers. In Fig. 4 the distributions of the log gaps have a faster finite-size convergence, than those of the gaps in Fig. 2. The curves in Fig. 4 are almost indistinguishable for larger sizes. In addition, we notice a difference between the shape of the numerical curves and the analytical results, the former being somewhat shifted to the right around zero. This shift indicates a reduction of the value of the gap due to small, but relevant correlations between the rare regions.

\section{Numerical test for uncorrelated Kesten variables}

To test the possible convergence of the finite-size corrections for uncorrelated variables we have repeated the analysis in the previous subsection for a parent distribution generated by Kesten random numbers. Kesten-type random variables are defined as [29]

$$
u_{m}=1+\sum_{i=1}^{m} \prod_{j=1}^{i} s_{j}+\cdots,
$$

where the $s_{j}$ 's are iid random numbers. It is known that in the limit of $m \rightarrow \infty$ there is a limit distribution $\rho_{\infty}(u)$, provided $[\ln s]_{\mathrm{av}}<0$. This limit distribution has a power-law tail

$$
\rho_{\infty}(u) \sim u^{-(1+\alpha)}, \quad u \gg 1,
$$



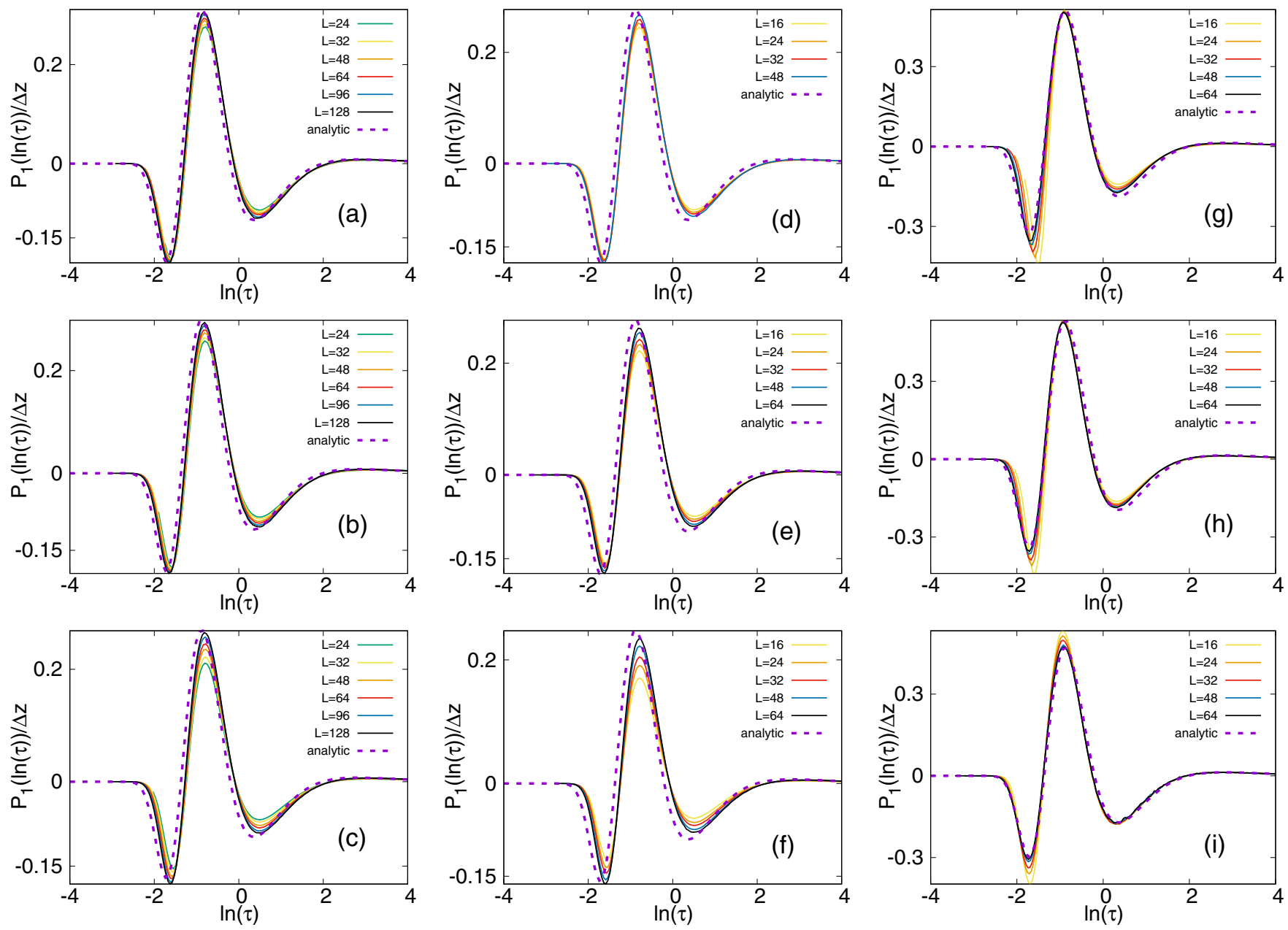

FIG. 4. The same as in Fig. 3 for the log variable and using the second standardization condition in Eq. (12).

where the exponent is the positive root $\alpha>0$ of the equation

$$
\left[s^{\alpha}\right]_{\mathrm{av}}=1 .
$$

Note that the relation for $\alpha$ is analogous to the equation for the dynamical exponent $z$ of the RTIM, see Eq. (5), and so we have the relation $\alpha=1 / z$. To have a direct relation with the RTIM calculations we set $s_{j}=J_{j} / h_{j}$ where $J_{j}$ and $h_{j}$ are taken from the distributions in Eq. (34). In the numerical calculation we have $m=64$, and considered the maximum of a set of $L=16,24,32,48$, and 64 Kesten numbers, denoted by $\tau$, so that we have $m \geqslant L$ in each case. We have checked that at such a value of $m$ the truncation of the series in Eq. (38) has negligible error.

We have analyzed the distribution of the maximum values at the three points $h_{0}=2, h_{0}=3$, and $h_{0}=4$ as done previously for the gaps of the RTIM. Using the first standardization condition in Eq. (11) the finite-size corrections are shown in the third column of Fig. 3. Here, the analytical results are obtained with the exponents $\gamma=z\left(h_{0}\right)$ and $\gamma^{\prime}=-1$, the latter following from the analytical results in Ref. [36]. It is seen in Fig. 3 that there is an overall good agreement between the numerical and analytical results. There is some size dependence of the corrections, which changes sign between $h_{0}=2$ and $h_{0}=3$. In comparison to the distribution of the gaps in the RTIM, the agreement with the analytical results is much better and the finite-size corrections are smaller.

We have repeated the analyses of the data by considering $\log$ variables, as given in Eqs. (31) and (32) and using the second standardization condition in Eq. (12). Results of the numerical analysis in this case are given in the third column of Fig. 4. As seen in this figure, there is some finite-size dependence in the scaled numerical data, but the expected asymptotic curves agree well with the analytical results. We note that practically no finite-size dependence of the correction term is observed for a pure power parent distribution in Fig. 1. This indicates that further correction to scaling terms in Eq. (29) could be responsible for the slower convergence in the case of Kesten variables.

\section{DISCUSSION}

In this paper, we have considered a paradigmatic model of random quantum magnets, the random transverse Ising model in 1D, and studied the distribution of low-energy excitations in the paramagnetic Griffiths phase, with extensive numerical methods. We have considered a large set of random samples $\left(10^{10}\right)$ and the calculation is performed by the approximate, but asymptotically correct SDRG method (up to $L=512$ ) and for comparison we also used the free-fermion 
method for shorter chains (up to a size $L=64$ ). Analyzing the distribution of the gaps, we have demonstrated with high precision that-in agreement with previous expectations- the limit distribution in the thermodynamic limit is in the Fréchet form. The Fréchet distribution depends on the value of the dynamical exponent $z$ and we have shown that a powerful method of calculation is through fitting a Fréchet curve to the numerical gap distributions. In this way, effective, finite-size estimates are obtained for the dynamical exponent $z_{L}$, which then are extrapolated to $L \rightarrow \infty$. According to the numerical data this convergence is in the form $z-z_{L} \sim \ln ^{\omega} L / L$, where the exponent of the logarithm $\omega$ depends on the distance from the critical point.

More interestingly, we have systematically studied the finite-size corrections to the limit law and showed that the difference between the numerical distribution and the asymptotic Fréchet form scales with $z-z_{L}$, and this scaled difference $P_{1}(\varepsilon ; z)$ is a unique function of the value of the gap $\varepsilon$. We have performed this type of analysis for the gap, using the first standardization condition in Eq. (11), as well as for the log gap, where the second standardization condition in Eq. (12) was used. In both cases, the asymptotic form of the scaled finite-size correction function are found similar (if not identical) for the data with the free-fermion calculation and that from the SDRG iteration.

We can thus conclude that the SDRG method provides realistic results about the gap probability distribution function in the Griffiths phase of the RTIM, which is manifested in the following observations. (i) The dynamical exponent in Eq. (5) is exactly given by SDRG, from the comparison of the exact treatment in Ref. [10] with those in Refs. [8,9]. (ii) The probability density of the gap is given in the Frechét form by analytic calculation of the SDRG in the vicinity of the critical point in the thermodynamic limit [32]. The validity of this result has been extended to the complete Griffiths phase by our numerical SDRG calculations. (iii) We have also calculated numerically by SDRG the finite-size corrections to the probability distribution function and demonstrated that they satisfy finite-size scaling properties. (iv) The numerical calculations are repeated through the free-fermion method and the scaled finite-size corrections are found very similar in the two cases. We have observed that the finite-size corrections represent a very sensitive test, so we propose that this perturbation term is correctly described by the SDRG method.

The measured finite-size correction functions are also compared with the analytical results of iid random numbers, having the same decay exponent $(\gamma=z$ for the gaps and $\gamma=0$ for the log gaps) and correction to scaling exponent $\gamma^{\prime}=-1$. The two curves are found to have similar shape, but there are also differences in the asymptotic forms. We have checked that the observed differences are larger than the statistical error of the calculation. For this purpose we have analyzed with identical methods the same set of uncorrelated Kesten random variables having the same characteristic exponent $z$. For these iid random numbers the asymptotic finite-size corrections are found to be well described by the analytical results.

The observed difference in the finite-size corrections between the numerical curves and the analytical iid results indicates that the weak correlations between low-energy excitations in the RTIM are relevant. This is likely related to the fact that the linear extension of regions with a large size $l$ scales as $l \sim \ln L$. Even if the dominant rare regions had a finite extent, as considered in Ref. [35], their possible positions in the chain are constrained by the other more extended clusters, potentially resulting in a logarithmic multiplicative factor in Eq. (37).

Our investigations have concluded on the RTIM in 1D, but the observed results are possibly valid for other random quantum systems as well with localized excitations. For example, we can mention the RTIM in higher dimensions [37-39], the random quantum Potts [40] and Ashkin-Teller models [41], and generally random quantum magnets with short range interactions and with discrete symmetry. Similar conclusions should apply to some nonequilibrium processes, such as to the random asymmetric exclusion process [42] and the random contact process [43] as well.

\section{ACKNOWLEDGMENTS}

This work was supported by the Hungarian Scientific Research Fund under Grants No. K128989 and No. KKP-126749 as well as by the Project GINOP-2.3.2-15-2016-00036 cofinanced by the European Social Fund and the Hungarian Government. F.I. is indebted to Katalin Ozogány for sending her Ph.D. thesis [33], and for valuable correspondence, as well as to L. Gránásy for useful discussions.

\section{APPENDIX A: METHODS TO CALCULATE THE GAP IN THE RTIM}

The energy scale of the model is given by the lowest excitation energy of the Hamiltonian in Eq. (1). This is calculated for finite chains by the asymptotically exact SDRG method through iteration and for shorter chains by free-fermion techniques.

\section{The SDRG method}

In the SDRG procedure [4] we perform consecutive decimation steps, each time considering the local excitations, say at position $i$. These excitations correspond to either couplings or sites, having the value of the associated gaps $2 J_{i}$ and $2 h_{i}$, respectively. These gaps are sorted in descending order and the largest one, denoted by $\Omega$, which sets the energy scale in the problem, is eliminated. Then, between remaining degrees of freedom, new terms in the Hamiltonian are generated through perturbation calculation. This procedure is successively iterated, during which $\Omega$ monotonously decreases. At the fixed point, with $\Omega^{*}=0$, one makes an analysis of the distribution of the different parameters and calculates the scaling properties. In the following we describe the elementary decimation steps.

\section{a. Strong-coupling decimation}

In this case, the largest local term in the Hamiltonian is a coupling, say $\Omega=J_{i}$, connecting sites $i$ and $i+1$ and the two-site Hamiltonian is given by

$$
\hat{H}_{c p}=-J_{i} \sigma_{i}^{z} \sigma_{i+1}^{z}-h_{i} \sigma_{i}^{x}-h_{i+1} \sigma_{i+1}^{x} .
$$


The spectrum of $\hat{H}_{c p}$ contains four levels, the lower two being separated from higher two by a gap of $\sim 2 J_{i}$. We omit the higher two levels, corresponding to merging the two strongly coupled sites into a spin cluster in the presence of a (renormalized) transverse field $\tilde{h}$, the value of which is given by second-order perturbation calculation

$$
\tilde{h}=\frac{h_{i} h_{i+1}}{J_{i}} .
$$

\section{b. Strong-transverse-field decimation}

In this case, the largest local term is a transverse field, say $h_{i}$, and due to its large value this site does not contribute to the longitudinal magnetization and therefore it is eliminated. The renormalized coupling between the remaining sites is given by

$$
\tilde{J}=\frac{J_{i-1} J_{i}}{h_{i}}
$$

which is calculated by second order perturbation method.

To calculate the smallest gap of a given sample $\varepsilon$, we perform $(L-1)$ decimation steps up to the last spin cluster having an effective transverse field $\tilde{h}=\varepsilon / 2$.

\section{Free-fermion technique}

In this method $\hat{H}$ is expressed in terms of spinless free fermions [26-28]. In the first step, the spin operators $\sigma_{i}^{x, y, z}$ are mapped to fermion creation (anni- hilation) operators $c_{i}^{\dagger}\left(c_{i}\right)$ by using the Jordan-Wigner transformation [26] $c_{i}^{\dagger}=a_{i}^{+} \exp \left[\pi \iota \sum_{j=1}^{i-1} a_{j}^{+} a_{j}^{-}\right]$and $c_{i}=$ $\exp \left[\pi \imath \sum_{j=1}^{i-1} a_{j}^{+} a_{j}^{-}\right] a_{i}^{-}$, where $a_{j}^{ \pm}=\left(\sigma_{j}^{x} \pm \imath \sigma_{j}^{y}\right) / 2$, and the Ising Hamiltonian in Eq. (1) is written in a quadratic form

$$
\hat{H}=-\sum_{i=1}^{L} h_{i}\left(2 c_{i}^{\dagger} c_{i}-1\right)-\sum_{i=1}^{L-1} J_{i}\left(c_{i}^{\dagger}-c_{i}\right)\left(c_{i+1}^{\dagger}+c_{i+1}\right) .
$$

In the second step, the Hamiltonian in Eq. (A4) is diagonalized through a canonical transformation [27], in terms of the new fermion creation (annihilation) operators $\eta_{k}^{\dagger}\left(\eta_{k}\right)$,

$$
\hat{H}=\sum_{k=1}^{L} \epsilon_{k}\left(\eta_{k}^{\dagger} \eta_{k}-\frac{1}{2}\right)
$$

The energies of free fermionic modes $\epsilon_{k}$ are given by the eigenvalues of a $2 L \times 2 L$ tridiagonal matrix

$$
T=\left(\begin{array}{ccccccc}
0 & h_{1} & & & & & \\
h_{1} & 0 & J_{1} & & & & \\
0 & J_{1} & 0 & h_{2} & & & \\
& & h_{2} & 0 & \ddots & & \\
& & & \ddots & \ddots & J_{L-1} & \\
& & & & J_{L-1} & 0 & h_{L} \\
& & & & & h_{L} & 0
\end{array}\right)
$$

and we consider only the $\epsilon_{k} \geqslant 0$ part of the spectrum [44]. The smallest gap of $\hat{H}$ in Eq. (1) is given by $\varepsilon=\min \left|\epsilon_{k}\right|$.
[1] R. B. Griffiths, Nonanalytic Behavior Above the Critical Point in a Random Ising Ferromagnet, Phys. Rev. Lett. 23, 17 (1969).

[2] B. McCoy, Incompleteness of the Critical Exponent Description for Ferromagnetic Systems Containing Random Impurities, Phys. Rev. Lett. 23, 383 (1969).

[3] T. Vojta, Rare region effects at classical, quantum and nonequilibrium phase transitions, J. Phys. A: Math. Gen. 39, R143 (2006).

[4] For reviews, see F. Iglói and C. Monthus, Strong disorder RG approach of random systems, Phys. Rep. 412, 277 (2005); Strong disorder RG approach - a short review of recent developments, Eur. Phys. J. B 91, 290 (2018).

[5] S.-K. Ma, C. Dasgupta, and C. K. Hu, Random Antiferromagnetic Chain, Phys. Rev. Lett. 43, 1434 (1979); C. Dasgupta and S.-K. Ma, Low-temperature properties of the random Heisenberg antiferromagnetic chain, Phys. Rev. B 22, 1305 (1980).

[6] D. S. Fisher, Random Transverse Field Ising Spin Chains, Phys. Rev. Lett. 69, 534 (1992); Critical behavior of random transverse-field Ising spin chains, Phys. Rev. B 51, 6411 (1995).

[7] D. S. Fisher, Phase transitions and singularities in random quantum systems, Physica A 263, 222 (1999).

[8] F. Iglói, R. Juhász, and P. Lajkó, Griffiths-McCoy Singularities in Random Quantum Spin Chains: Exact Results Through Renormalization, Phys. Rev. Lett. 86, 1343 (2001).

[9] F. Iglói, Exact renormalization of the random transverse-field Ising spin chain in the strongly ordered and strongly disordered Griffiths phases, Phys. Rev. B 65, 064416 (2002).
[10] F. Iglói and H. Rieger, Anomalous diffusion in disordered media and random quantum spin chains, Phys. Rev. E 58, 4238 (1998).

[11] J. C. Getelina, F. C. Alcaraz, and J. A. Hoyos, Entanglement properties of correlated random spin chains and similarities with conformal invariant systems, Phys. Rev. B 93, 045136 (2016).

[12] M. J. Thill and D. A. Huse, Equilibrium behavior of quantum Ising spin-glass, Physica A 15, 321 (1995).

[13] R. Juhász, Y.-C. Lin, and F. Iglói, Strong Griffiths singularities in random systems and their relation to extreme value statistics, Phys. Rev. B 73, 224206 (2006).

[14] R. A. Fisher and L. H. C. Tippett, Limiting forms of the frequency distribution of the largest or smallest member of a sample, Proc. Cambridge Philos. Soc. 24, 180 (1928).

[15] E. J. Gumbel, Statistics of Extremes (Dover, New York, 1958).

[16] B. V. Gnedenko, Sur La Distribution Limite Du Terme Maximum D'Une Serie Aleatoire, Ann. Math. 44, 423 (1943).

[17] W. Weibull, A statistical distribution function of wide applicability, J. Appl. Mech. 18, 293 (1951).

[18] J. Galambos, The Asymptotic Theory of Extreme Order Statistics (Wiley, New York, 1978).

[19] M. R. Leadbetter, G. Lindgren, and H. Rootzén, Extremes and Related Properties of Random Sequences and Processes (Springer, New York, 2012),

[20] S. Resnick and L. de Haan, Second-order regular variation and rates of convergence in extreme-value theory, 
Ann. Probab. 24, 97 (1996); L. de Haan and U. Stadtmüller, Generalized regular variation of second order, J. Austral. Math. Soc. 61, 381 (1996).

[21] G. Györgyi, N. R. Moloney, K. Ozogány, and Z. Rácz, FiniteSize Scaling in Extreme Statistics, Phys. Rev. Lett. 100, 210601 (2008).

[22] G. Györgyi, N. R. Moloney, K. Ozogány, Z. Rácz, and M. Droz, Renormalization-group theory for finite-size scaling in extreme statistics, Phys. Rev. E. 81, 041135 (2010).

[23] E. Bertin and G. Györgyi, Renormalization flow in extreme value statistics, J. Stat. Mech. (2010) P08022.

[24] L. Zarfaty, E. Barkai, and D. A. Kessler, Accurately approximating extreme value statistics, J. Phys. A: Math. Theor. 54, 315205 (2021).

[25] S. N. Majumdar, A. Pal, and G. Schehr, Extreme value statistics of correlated random variables: A pedagogical review, Phys. Rep. 840, 1 (2020).

[26] P. Jordan and E. Wigner, Über das Paulische Äquivalenzverbot, Z. Phys. 47, 631 (1928).

[27] E. Lieb, T. Schultz, and D. Mattis, Two soluble models of an antiferromagnetic chain, Ann. Phys. (NY) 16, 407 (1961); S. Katsura, Statistical mechanics of the anisotropic linear heisenberg model, Phys. Rev. 127, 1508 (1962); P. Pfeuty, The one-dimensional Ising model with a transverse field, Ann. Phys. (Paris) 57, 79 (1970).

[28] P. Pfeuty, An exact result for the 1D random Ising model in a transverse field, Phys. Lett. 72A, 245 (1979).

[29] H. Kesten, Random difference equations and renewal theory for products of random matrices, Acta Math. 131, 208 (1973).

[30] A. P. Young and H. Rieger, Numerical study of the random transverse-field Ising spin chain, Phys. Rev. B 53, 8486 (1996).

[31] F. Iglói and H. Rieger, Random transverse Ising spin chain and random walks, Phys. Rev. B 57, 11404 (1998).

[32] D. S. Fisher and A. P. Young, Distributions of gaps and endto-end correlations in random transverse-field Ising spin chains, Phys. Rev. B 58, 9131 (1998).

[33] K. Ozogány, Ph.D. thesis, Loránd Eötvös University, 2012.

[34] V. Privman, Universal size dependence of the free energy of finite systems near criticality, Phys. Rev. B 38, 9261 (1988).

[35] T. Vojta and J. A. Hoyos, Criticality and Quenched Disorder: Rare Regions vs. Harris Criterion, Phys. Rev. Lett. 112, 075702 (2014).
[36] C. de Calan, J. M. Luck, Th. M. Nieuwenhuizen, and D. Petritis, On the distribution of a random variable occurring in 1D disordered systems, J. Phys. A 18, 501 (1985).

[37] O. Motrunich, S.-C. Mau, D. A. Huse, and D. S. Fisher, Infinite-randomness quantum Ising critical fixed points, Phys. Rev. B 61, 1160 (2000); Y.-C. Lin, N. Kawashima, F. Iglói, and H. Rieger, Numerical renormalization group study of random transverse Ising models in one and two space dimensions, Progress Theor. Phys. 138, 479 (2000); D. Karevski, Y.-C. Lin, H. Rieger, N. Kawashima, and F. Iglói, Random quantum magnets with broad disorder distribution, Eur. Phys. J. B 20, 267 (2001); Y.-C. Lin, F. Iglói, and H. Rieger, Entanglement Entropy at Infinite Randomness Fixed Points in Higher Dimensions, Phys. Rev. Lett. 99, 147202 (2007); R. Yu, H. Saleur, and S. Haas, Entanglement entropy in the two-dimensional random transverse field Ising model, Phys. Rev. B 77, 140402(R) (2008).

[38] I. A. Kovács and F. Iglói, Critical behavior and entanglement of the random transverse-field Ising model between one and two dimensions, Phys. Rev. B 80, 214416 (2009); Renormalization group study of the two-dimensional random transverse-field Ising model, ibid. 82, 054437 (2010).

[39] I. A. Kovács and F. Iglói, Infinite disorder scaling of random quantum magnets in three and higher dimensions, Phys. Rev. B 83, 174207 (2011); Renormalization group study of random quantum magnets, J. Phys. Condens. Matter 23, 404204 (2011).

[40] T. Senthil and S. N. Majumdar, Critical Properties of Random Quantum Potts and Clock Models, Phys. Rev. Lett. 76, 3001 (1996).

[41] E. Carlon and P. Lajkó, and F. Iglói Disorder Induced Crossover Effects at Quantum Critical Points, Phys. Rev. Lett. 87, 277201 (2001).

[42] R. Juhász, L. Santen, and F. Iglói, Partially Asymmetric Exclusion Models with Quenched Disorder, Phys. Rev. Lett. 94, 010601 (2005).

[43] J. Hooyberghs, F. Iglói, and C. Vanderzande, Strong Disorder Fixed Point in Absorbing-State Phase Transitions, Phys. Rev. Lett. 90, 100601 (2003); Absorbing state phase transitions with quenched disorder, Phys. Rev. E 69, 066140 (2004).

[44] F. Iglói and L. Turban, Common Trends in the Critical Behavior of the Ising and Directed Walk Models, Phys. Rev. Lett. 77, 1206 (1996). 